\title{
Digital Technology in English Education: Linguistic Gain and Pain Points
}

\author{
Budsaba Kanoksilapatham
}

\begin{abstract}
Digital technology has recently become increasingly essential in all aspects of life, including education. The outbreak of COVID-19, which has now been declared a pandemic, has intensified and amplified the use of digital technology. However, the impacts of digital technology integrated learning have not yet been thoroughly investigated. The objectives of this study are twofold: to assess the impacts of incorporating digital technology into English education with regard to Thai university students' linguistic gain and to identify pain points in online learning. Initially, a pretest was developed and administered to first year university students majoring in humanities. Subsequently, the participants were exposed to ten sets of strategically selected grammatical online lessons representing ten grammatical points for 19 days, during which time they were free to explore and exploit the lessons. Then, toward the end of the exposure, a parallel posttest was administered, followed by a post online lesson exposure questionnaire and a semi-structured interview with randomly selected students. The data analysis revealed that, while the participants' linguistic abilities improved significantly, they appeared to have poor learning management. Therefore, although digital technology is valuable and beneficial, some learners need to be scaffolded before embarking on the digital technology integrated English education journey.
\end{abstract}

Index Terms-Digital technology, English education, grammar, university, Thailand.

\section{INTRODUCTION}

Digital technology is known to refer to electronic tools, systems, devices, and resources that generate, store, or process data. Some of the familiar examples include social media, online games, multimedia, and mobile phones. In fact, digital technology has recently become increasingly essential in all aspects of life, including education. The outbreak of COVID-19, and now the pandemic, has intensified and amplified the use of digital technologies in education, resulting in pervasive digital learning. In fact, digital technology in English language education can vary greatly in terms of its characteristics and potential. For instance, some are tools that provide a collection of texts that learners can passively enjoy reading, whereas others provide language tasks and activities that allow for not only a more personalized but also interactive learning approach.

The COVID-19 outbreak, and now pandemic, has exerted

Manuscript received September 9, 2021; revised November 1, 2021. This study was financially supported by Silpakorn University through the Faculty of Arts. My heart-felt appreciation goes to the Dean of the Faculty of Arts, Associate Professor Warangkana Nibhatsukit, $\mathrm{PhD}$, who entrusted me with the task of initiating and leading this research project.

Budsaba Kanoksilapatham is with the Faculty of Management Science, Silpakorn University, Thailand (e-mail: kanoksib@hotmail.com). one of the most significant global impacts on education. With a focus on the Thai context, the outbreak in 2019 swept such a large area at such a rapid pace that Thai universities were prescribed to provide online learning and teaching in an overnight time. Without preparation for this drastic shift to switch to online learning and teaching, digital technology has become a valuable asset and resource that should be used in order not to sabotage learning and teaching activities. At this juncture, it is worth noting that in a variety of contexts, across education levels and language skills, and via a variety of platforms and devices, several academics have endorsed the positive impacts of incorporating digital technology in language education [1]-[5]. However, scant attention has been paid to the investigation of online learning's impacts particularly on higher education in Thailand. To be more specific, it is unclear whether Thai students have benefited from online learning in English education. Furthermore, it is unknown whether Thai learners struggled with online lessons. Given the potential that the COVID-19 pandemic will be with us in the foreseeable future, the answers or insights relevant to these questions will contribute to the learners' enhanced learning outcomes and more effective teachers' pedagogy.

\section{METHODS}

A mixed methods research design was employed, which included both quantitative and qualitative elements. Therefore, this section is divided into a number of subsections, describing individual methodological aspects pertinent to the current study.

\section{A. Digital Technology Subcategorized}

With the focus on English education, digital technology is subcategorized in this study as online lessons which in turn refer to freely accessible and openly licensed materials and resources or commonly known as open educational resources (henceforth OER). Inherent advantages of OER online lessons are numerous, including readiness for use, a wide range of topics, language skills and levels, high volume, and free use.

\section{B. Research Questions}

Two research questions were addressed in this study: 1) what are the learning outcomes of Thai university students after being exposed to grammar online lessons, and 2) what are the students' difficulties as far as online learning is concerned?

\section{Context and Participants}

A medium sized public university in central Thailand was 
chosen to provide a context for this study. Given that English is compulsory for first-year students, and students in the humanities are more likely to be interested in English than those in the sciences, the target participants of this study were initially a pool of 600 first-year university students in the humanities who were enrolled in the course of English for international communication, whose age ranged from 18 to 21 years old.

\section{Online Lessons: Scale and Scope}

This study is aimed at examining the role of digital technology in English education. English education, on the other hand, encompasses the teaching and learning a wide range of language skills, including listening, speaking, reading, pronunciation, vocabulary, and writing. Due to the numerous subfields of English education, this paper will concentrate on English grammar learning.

Unlike other language skills, the role of English grammatical skills can be considered a very interesting debate. Whereas many scholars consider that grammatical knowledge is not so important and should not be emphasized in class, several renowned scholars have strongly advocated that grammar teaching and learning cannot be underestimated [6]-[12]. According to these scholars, grammar knowledge is of paramount importance in communication. That is, grammar facilitates understanding of the target language in an interesting way, allowing learners to use it precisely, productively, and creatively. Additionally, grammar knowledge also lends support for learners to be more observant and sensitive when learning different languages, recognizing similarities and differences across languages. To further support this standpoint, for example, Swan [11], [13] states several reasons for prioritizing and highlighting grammar instruction, including the fact that it is always present, quite systematic, testable, and contributes to comprehensibility and social acceptability.

In the context of Thailand, teaching and testing are inextricably linked. Grammar, of course, is one of the test constructs assessed across the entire educational paradigm and thus has a stronghold in any English curriculum. However, despite the emphasis on grammar, Thai learners do not appear to master grammar knowledge. For instance, a relatively recent study [14] conducted at a university demonstrated that graduate students agree that grammar is essential in communication. Nevertheless, their grammar test scores, in both recognition and production tests, are unsatisfactory.

Similar to other language skills, grammar in turn encompasses a number of subskills. To ensure that the grammar subskills focused in this study are truly essential and relevant to our first-year students, commercial textbooks used in this university's English foundation courses were scrutinized to identify grammar subskills to be included in the study. As a result, ten subskills were identified as commonly included and typically sequenced in the commercial textbooks as follows: tenses, adjectives \& adverbs, comparisons, articles, modal verbs, infinitives \& gerunds, passive sentences, conditionals, relative clauses, and reported speech. These subskills were subsequently used as key words to search the Internet for relevant OER links.

\section{E. OER Link Searching and Validating}

Based on each of the ten grammar subskills identified in the previous section, corresponding OER links were sought on the Internet. As expected, multiple links were produced from the search. However, it is possible that certain online lesson materials, without oversight from experts and authorities or input from academic grammarians, are likely to contain mistakes (e.g., typos, grammatical errors) and might not be appropriate to the students' level (e.g., difficulty level, language use, and content). As a result, the links obtained from the initial search were subsequently validated by three experts who have extensive experience in teaching English at the university level. Two of them are English native speakers, and the other is a Thai faculty member with at least six years and more than 20 years of English teaching experience, respectively. The OER links obtained through an Internet search underwent validation in terms of content, difficulty level, and appropriateness. In short, each of the ten grammar subskills was represented by two selected and subsequently validated OER links, yielding a total of 20 OER links.

\section{F. Instruments Developed}

The instruments of this study consist of a personal information sheet, a pretest and a parallel posttest focusing on ten grammar subskills, a post OER exposure questionnaire, and a set of semi-structured interview questions. These instruments were developed to serve a variety of purposes. First, a personal information sheet was to elicit individual students' personal information in order to create accounts for them on the university's MOODLE system. Second, a grammar pretest and a parallel posttest were used not only to assess the learning outcomes but also to compare the students' grammar knowledge both before and after exposure to the online lessons. Both of the pretest and the parallel posttest consist of 40 multiple choice items each (four items with four alternatives for each grammar subskill). To illustrate, Fig. 1 presents two examples of corresponding pretest and posttest items on the grammar subskills of relative clauses (item no. 1) and articles (item no. 2).

\begin{tabular}{|c|c|}
\hline Pretest & Posttest \\
\hline $\begin{array}{l}\text { 1. Lopburi, is located in the } \\
\text { central region of Thailand, used to } \\
\text { be the capital city in the Ayudhaya } \\
\text { Period. } \\
\text { 1. where } \\
\text { 2. that } \\
\text { 3. which } \\
\text { 4. in which }\end{array}$ & $\begin{array}{l}\text { 1. Venice, } \text { is my favorite } \\
\text { city, is one of the most popular } \\
\text { tourist destinations in Italy. } \\
\text { 1. where } \\
\text { 2. that } \\
\text { 3. which } \\
\text { 4. in which }\end{array}$ \\
\hline $\begin{array}{l}\text { 2. It is known that } \_ \text {dolphins are } \\
\text { one of the most intelligent animals } \\
\text { on _ earth. } \\
\text { 1. no article, the } \\
\text { 2. the, no article } \\
\text { 3. no article, no article } \\
\text { 4. the, the }\end{array}$ & $\begin{array}{l}\text { 2. It is well established that } \\
\text { blue whales are the largest } \\
\text { mammals in } \\
\text { 1. no article, the } \\
\text { 2. the, no article } \\
\text { 3. no article, no article } \\
\text { 4. the, the }\end{array}$ \\
\hline
\end{tabular}

Fig. 1. Parallel pretest and posttest items.

To examine the participants' learning management and online learning behaviors, a post OER exposure questionnaire consisting of four questions was developed (1. 
the average access hours per day, 2. the preferred time period for the access, 3. the devices used to access the lessons, and 4. the manner in which the access was made - accessing either all of the OER lessons provided or partly).

Finally, a set of three semi-structured interview questions was devised to explore and delve further into their online learning exposure. Questions 1 and 2 are about the students' attitudes toward the OER exposure and the strength/weakness of the online exposure, respectively, whereas question 3 on their preferred English lesson delivery mode $(100 \%$ on site or traditional classroom setting, $100 \%$ online learning, or hybrid with on site and online integration). It should be noted that, in this study, all of the instruments employed underwent validation performed by the same three experts as previously described.

\section{PROCEDURES}

This section comprises two principal subsections: data collection and data analysis.

\section{A. Data Collection}

All English teachers teaching the first-year English course were requested to share the details of the research project with the pool of approximately 600 students in their English classrooms. Additional emphasis was made to remind the students that their participation was entirely voluntary, and it thus had no connection with the courses they were enrolled in at the time of study.

A total of 356 students volunteered to participate and attended our initial face-to-face meeting on campus. In this meeting, the project was presented in detail, which was followed by the completion of the participants' profile questionnaire in Thai (hard copy) so that corresponding MOODLE accounts could be generated. Next, the 40-item grammar pretest was administered, with a maximum time allocation of 30 minutes. This initial meeting was purposefully scheduled during the final week of the semester. This means that following the meeting, the students were preoccupied with their two-week final examinations.

After the examinations was a three-week semester break. During the break, the 356 registered students were supposed to freely access and explore the grammar OER links which were made available 24 hours for a period of 19 days. This means that they had complete freedom with regard to OER lesson access time, pace, length, and manner (intermittently or solidly). In order to ensure that their OER access experience was a smooth and pleasant one, a help desk under the researcher's supervision was available in case they encountered difficulties or required additional assistance. A few days prior to Day 19, the registered participants were notified of the impending posttest, which was made available to access for three days. They could access the 40-item posttest whenever they wanted to, but only once for a maximum of 30 minutes.

Following the posttest, the participants were requested to complete the post OER exposure questionnaire, which consists of four questions previously described. The last procedure implemented was semi-structured interviewing. Ten registered students were randomly selected to participate in semi-structured interviews conducted over the phone or other devices based on the students' convenience and preference. A total of ten interview sessions of about 15 minutes each were recorded and transcribed. All data collected in this study (the pretest and posttest scores, the post OER questionnaire data, and the interview data) were recorded in the MOODLE system for subsequent analysis.

\section{B. Data Analysis}

Out of 365 students who registered for the pretest, according to the MOODLE system record, only 189 students completed the posttest. As a result, only the pretest and posttest scores of the corresponding 189 students, as well as the post OER questionnaire data, were statistically analyzed. At this point, the pretest and posttest scores were calculated and compared using a t-test to determine whether their pretest and posttest scores were significantly different. Content analysis was performed to categorize the interview data into emerging themes.

\section{RESUlts}

The current study aims to examine the impacts of digital learning on Thai university students' English grammar learning outcomes as well as any difficulties encountered during their online learning experience. The results of the analyses of the three datasets are presented individually in the subsections that follow.

\section{A. Pretest and Posttest Score Analysis Results}

Fig. 2 displays the pretest and posttest mean scores pertaining to individual grammar subskills, which are represented by four items or four points each. The pretest mean scores for the ten grammar subskills range from 1.83 to 3.68 , while the posttest mean scores range from 2.56 to 3.74 .

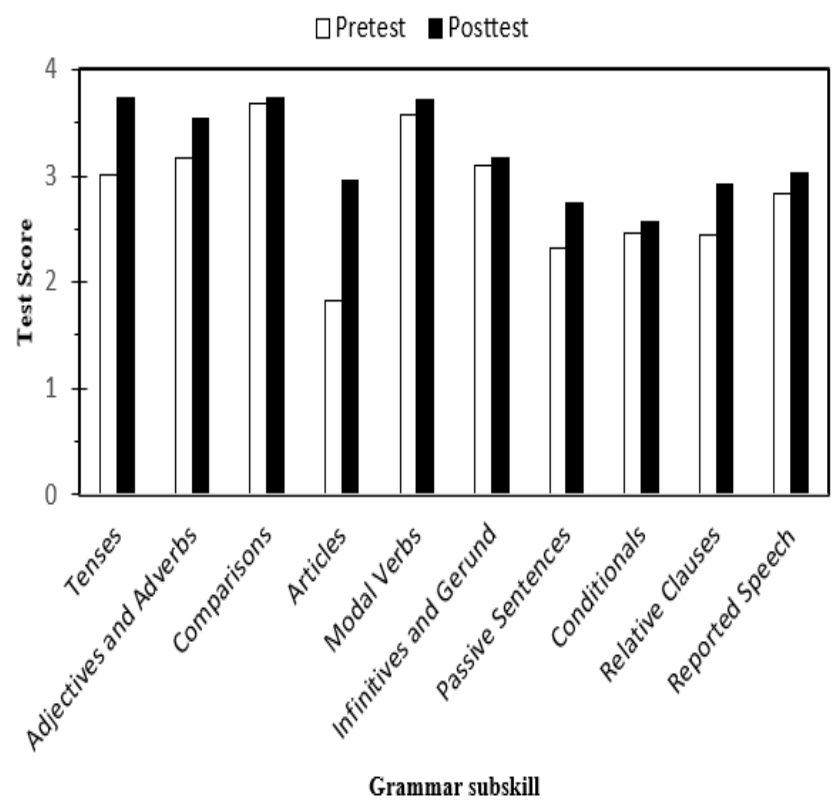

Fig. 2. Pretest and posttest scores of grammar subskills.

In scrutiny, the students' strongest skill in the pretest was comparisons, with the highest mean score of 3.68, and the subskill of articles the weakest with the mean score of 1.83 . 
However, the picture does not become completely identical when the posttest scores were considered. The comparisons subskill, like the pretest scores, did not appear to pose significant difficulty to the students, with the highest mean posttest score of 3.74 ; conditionals, on the other hand, appeared to be the most difficult for the students, with the lowest mean posttest score of 2.56. It should be noted that, with only four items representing each subskill, generalizations must be interpreted with caution. In addition, this research focuses on the impacts of online learning on grammar skills in general. Therefore, these findings remain tentative and need to be substantiated by subsequent studies. In a broad sense, focusing on the entire grammar test, the posttest mean score of 32.08 is higher than that of the pretest of 28.41 (not shown). The comparison of the pretest mean score of the 189 participants $(M=28.41, S D=5.28)$ compared to the posttest mean score $(M=32.08, S D=6.12)$ demonstrated that the online lesson exposure contributed significantly to their grammar knowledge, $t(188)=5.76, p$ $<.05$. Based on these findings, it is possible to conclude that the students' exposure to OER appeared to contribute positively and substantially to their grammar knowledge. In other words, OER online lessons seem to exert a positive linguistic impact on the learners.

\section{B. Post OER Questionnaire Data Analysis Results}

As previously described, this questionnaire aims to elicit four pieces of information concerning the students' online learning behaviors and management, which will be elaborated on below.

To obtain information regarding the average daily number of hours they accessed the OER lessons, with the provision of five options (less than an hr, 1-2 hrs, 2-3 hrs, 3-4 hrs, more than $4 \mathrm{hrs}$ ), most of the respondents (41.27\%) spent 1-2 hours per day on the online lessons, followed by less than an hour (38.10\%); few participants (15.34\%) spent 2-3 hours, and very few $(2.65 \%$ each) $3-4$ hours and more than 4 hours per day. This finding is quite intriguing because it suggests that despite the fact that the OER lessons are generally interactive in nature and were carefully selected, they did not appear to be able to hold the students'attention for an extended period of time. In fact, it can be concluded that approximately $80 \%$ of the participants (with $41.27 \%$ and $38.10 \%$ combined) were unable to stay online for longer than 2 hours.

The second question in this section concerns the times of day when the students accessed the OER lessons. With four options available (1.01-6.00, 6.01-12.00, 12.01-18.00, 18.01-24.00), the majority of the respondents opted for $12.01-18.00$ (49.09\%), 6.01-12.00 (34.39\%), with the much smaller numbers for 1.01-6.00 and 18.01-24.00 of only $8.99 \%$ and $7.41 \%$, respectively.

Regarding the devices that the students used to access the online lessons, most of them used PCs (121 participants or $64.02 \%$ ), smartphones (60 participants or $31.75 \%$ ), and tablets/iPads ( 8 participants or $4.23 \%$ ). The final question of this questionnaire is whether the students accessed all of the online lessons during the 19-day period. Unfortunately, only $23.81 \%$ or 45 participants did, whereas the majority of the participants (144 participants or $76.19 \%$ ) did not.

\section{Interview Data Analysis Results}

The interview data were obtained from ten interview sessions conducted individually on the phone or other social media platforms. There are three interview questions. In the first place, when it came to the students' attitudes toward the OER online lessons, all of the ten interviewees were unanimously positive, strongly agreeing that the online lesson content was appropriate for their level and beneficial for their linguistic development.

The second question aims to elicit what the students perceived as strengths and weaknesses of the online lessons, based on their OER exposure. In this regard, most of the interviewees agreed that academically, or more precisely, linguistically, the online lessons improved their grammar knowledge, as evidenced by their higher scores in the posttest. Nevertheless, some disadvantages of online learning were reported by some interviewees. Some of them felt a bit incompetent when taking online tests because they were unfamiliar with the relatively novel test item display and had to engage in synchronous multi-tasking of selecting answers and scrolling up and down in case the answers needed to be checked and changed. To make the scenario worse, they encountered a not so complacent physical environment. To be precise, they were distracted by events or incidents in their home. Being present at home and studying online did not provide them with valid excuses when other family members inadvertently interfered with their online learning.

The final question asked during the interviews was what they decided when given three options for classroom delivery modes: $100 \%$ online, $100 \%$ on site, and hybrid learning. Surprisingly, they all advocated for the hybrid learning mode. They developed an understanding of what online learning could and could not provide as a result of their exposure to online lessons. One of the interviewees stated unequivocally that he was feeling lonely. Furthermore, they all admitted that, despite all the merits of online learning, they yearned for a catalyst agent to propel them to academic success - a language teacher. They all acknowledged the crucial role of a teacher as a person with some authority to ensure things go as planned or, in this case, to accomplish what the online lessons are entitled to. The interviewees added that the teacher presence would propel them to be more disciplined and responsible. They thus want to be able to attend a physical classroom so that they can interact with the teacher while taking online lessons.

\section{DISCUSSION}

The purpose of this study was to investigate the impacts of digital technology manifested through the implementation of OER online lessons on Thai university students' grammar knowledge. Furthermore, the purpose of this research is to investigate the students' learning difficulties as they relate to online lesson exposure. The results for each objective will be discussed in turn in this section.

With reference to the first objective, based on the quantitative analysis of the pretest and the parallel posttest scores, it is evident that 189 students participating in this study substantially improved their grammar knowledge. In 
short, linguistic gain is evident, which is consistent with previous research on Croatian university students using OER, Malaysian and Australian university students using social networking, and Korean university students using YouTube, conducted by Krelja Kurelovic [15], Hamid et al. [16], and Kim and Kim [17], respectively. One factor that may have contributed to this significant linguistic gain is that the OER lessons included in this study focused on specific grammar subskills. Furthermore, the OER links were painstakingly chosen from a wide range of Internet sources based on content, difficulty level, and appropriateness.

The second research objective addressed in this study is to examine the students' learning behaviors and management while exposed to the OER lessons in order to determine the learners' difficulties related to the online lesson access. Based on the analysis of the post OER questionnaires and the semi-structured interview accounts, certain findings need to be highlighted. First, only 189 from 356 students participated in the posttest, suggesting an alarming attrition rate of $47 \%$. Moreover, only a small number of the students managed to access all of the OER links provided (about $24 \%$ ). The decline in the number of the participants in the final pool, together with the questionnaire and interview data, indicate that engaging online lesson content is not the sole factor that maintains their attention or keeps them engaged and focused.

Taking into consideration their preference for a hybrid learning mode, the mystery can be unveiled. The OER lessons included in this study are interactive in nature. A close examination of what is missing from these online lessons and what the students yearn for a teacher in a physical classroom culminates in a conclusion that human interaction, be it teacher-student or student-student interaction, is unavailable in online learning. In fact, interaction is a crucial element in facilitating learning processes and enhancing learning outcomes [18], [19]. The absence of peer interaction and subsequent collaboration made one of the interviewees felt lonely during the online lessons. Without an opportunity to interact or getting support and encouragement from his peers or teachers, the students felt it was hard for them to stay focused.

Another impediment pertinent to the limited engagement and preference for hybrid classrooms appears to be the unanticipated switch to online learning mode triggered by the COVID-19 outbreak and pandemic in 2019 and 2020, respectively. The situation deteriorated and intensified so quickly to the point where online learning was the only option for dealing with the global crisis. Without prior preparation and warning, online classroom policies were implemented overnight. Indeed, English education in Thailand has supported the adoption of a communicative language teaching approach as well as the promotion of learner autonomy. However, in many academic settings, Thai teachers of English generally play a key role in planning activities and monitoring students' language learning progress. Learners were thus not fully prepared to be independent in, and responsible for, systematically managing their time, as well as monitoring and tracking their learning progress.

As this study has proved, digital technology is valuable and advantageous. Some students, however, require scaffolding before embarking on their digital technology-integrated English education journey. They may, for example, need to be trained how to exercise initial control over their physical learning environment. That is, they must be able to make effective use of the tools and OER resources accessible to them. Furthermore, when confronted with a large number of online lessons and programs, the students require scaffolding that will conceptually help them to prioritize the lessons available so that they can feel accomplished - learning what they do not understand. Finally, strategic scaffolding can be extremely beneficial in empowering students to make their own decisions and take charge of their own actions in order to attain their learning objectives.

In addition to the linguistic gain and pain points pertaining to online learning presented above, other findings generated from this study could be pedagogically beneficial. For instance, according to this study, the majority of the participants (about $80 \%$, with $41.27 \%$ and $38.10 \%$ combined) could not stay online for longer than 2 hours. Interestingly, a series of two studies conducted in 2020 and 2021 on the average time people around the world accessed social media each day [20], [21] showed that Thais spent approximately 3 hours each day on social media ( 2 hours 55 minutes in 2020, and 2 hours 48 minutes in 2021). Meanwhile, the global record shows that people in general spent 2 hours and 24 minutes on social media in total. Based on the data, it can thus be assumed that the impacts of digital technology for entertainment and for instruction can be different as far as learners' engagement is concerned. Furthermore, as revealed by this study, the students' preference for accessing online lessons in the afternoon is pedagogically applicable, implying to educators or practitioners that academic online activities scheduled in the morning, for example, may not be as successful as those scheduled in the afternoon. All these findings taken together can inculcate crucial pedagogical implications regarding class time or management for learners' maximum benefits.

\section{CONCLUSION}

There are currently a great variety of digital technologies that can be potentially exploited to enhance learning outcomes. This paper provided empirical evidence demonstrating that OER lessons, as one of the digital technology manifestations, can be one of the potentially promising tools to foster language learning in higher education, if carefully and systematically selected and compiled. However, as discussed earlier, pain points or hindrances pertinent to online lessons cannot be overlooked either. Therefore, OER lessons, as expressed by the students, should be integrated into the traditional learning environment to maximize learning outcomes. That is, the inherent challenges pertaining to online lessons can be mitigated by incorporating conventional classroom teaching. However, for this successful integration to be successful, a number of factors need to be considered, including preparing learners to be more responsible for their own learning, monitoring their success, and tracking their learning progress. They should be taught to be independent in making decisions and choosing 
appropriate learning and time management strategies regarding what they need to learn and in what order. In short, this study has provided us with a better understanding and insight into the employment of OER lessons in informing teaching practice in higher education in Thailand. It should be noted that the findings of this research may not be easily generalizable to other contexts of English education. In addition, future studies could broaden the scope to include multiple language skills and observe how the OER lessons impact individual skills. While this study focuses on receptive grammar skills, it remains to be investigated if this specific linguistic gain will be transferred to productive language skills in their actual linguistic performance.

\section{CONFLICT OF INTEREST}

The authors declare no conflict of interest of this submitted work.

\section{AUTHOR CONTRIBUTIONS}

Budsaba Kanoksilapatham conducted the research, analyzed the data, and wrote the paper.

\section{ACKNOWLEDGMENT}

I would like express my heartfelt appreciation to the 189 students who participated in this project.

\section{REFERENCES}

[1] N. A. Bakar, H. Latif, and A. Ya'acob, "Fusion of technology with language learning: Blog community," The Southeast Asian Journal of English Language Studies, vol. 23, no. 4, pp. 200-211, 2017.

[2] X. B. Chen, "Tablets for informal language learning: Student usage and attitudes," Language Learning \& Technology, vol. 17, no. 1, pp. 20-36, 2013.

[3] A. Chik, "Digital gaming and language learning: Autonomy and community," Language Learning and Technology, vol. 18, no. 2, pp. 85-100, 2014.

[4] D. Chun, R. Kern, and B. Smith, "Technology in language use, language teaching, and language learning," The Modern Language Journal, vol. 100, no. S1, pp. 64-80, 2016.

[5] L. Lee, "Learners' perceptions of the effectiveness of blogging for L2 writing in fully online language courses," International Journal of Computer-Assisted Language Learning and Teaching, vol. 7, pp. 19-33, 2017.

[6] A. Leis, A. Tohei, and S. D. Cooke, "Smartphone assisted language learning and autonomy," International Journal of Computer-Assisted Language Learning and Teaching (IJCALLT), vol. 5, no. 3, pp. 75-88, 2015.

[7] M. Celce-Murcia, "The importance of the discourse level in understanding and teaching English grammar," Teaching English Grammar to Speakers of Other Languages, pp. 3-18, 2016.

[8] P. K. Debata, "The importance of grammar in English language teaching - A reassessment," Language in India, vol. 13, no. 5, 482-486, 2013.

[9] E. Hinkel, "Prioritizing grammar to teach or not to teach: A research perspective," Handbook of Research in Second Language Teaching and Learning, pp. 369-383, 2016.
[10] M. Swan, "Seven bad reasons for teaching grammar-and two good ones," Methodology in Language Teaching: An Anthology of Current Practice, pp. 148-152, 2002.

[11] M. Swan and C. Walter, "Comments on R. Ellis's" current issues in the teaching of grammar: An SLA perspective": Readers Respond" Teach the Whole of the Grammar"," TESOL Quarterly, vol. 40, no. 4, pp. 837-839, 2006.

[12] F. Wang, "The necessity of grammar teaching," English Language Teaching, vol. 3, no. 2, pp. 78-81, 2010.

[13] M. Swan, "Cambridge grammar of English," ELT Journal, vol. 61, no. 1, pp. 75-78, 2007.

[14] S. Saengboon, "English grammar and Thai university students: An insurmountable linguistic battle?" English Language Teaching, vol. 10, no. 11, pp. 22-36, 2017.

[15] E. K. Kurelovic, "Advantages and limitations of usage of open educational resources in small countries," International Journal of Research in Education and Science, vol. 2, no. 1, pp. 136-142, 2016.

[16] S. Hamid, J. Waycott, S. Kurnia, and S. Chang, "Understanding students' perceptions of the benefits of online social networking use for teaching and learning," The Internet and Higher Education, vol. 26, pp. $1-9,2015$.

[17] S. Kim and H. C. Kim, "The benefits of YouTube in learning English as a second language: A qualitative investigation of Korean freshman students' experiences and perspectives in the US," Sustainability, vol. 13, no. 13, p. 7365, 2021.

[18] K. Johnson, An Introduction to Foreign Language Learning and Teaching, Routledge, 2017.

[19] O. Sert, "Classroom interaction and language teacher education," The Routledge Handbook of English Language Teacher Education, pp. 216-238, Routledge, 2019.

[20] N. Mahittivanicha, "Social media in 2020," 2020.

[21] N. Mahittivanicha, "Social media in 2021," 2021.

Copyright $(2022$ by the authors. This is an open access article distributed under the Creative Commons Attribution License which permits unrestricted use, distribution, and reproduction in any medium, provided the original work is properly cited (CC BY 4.0).

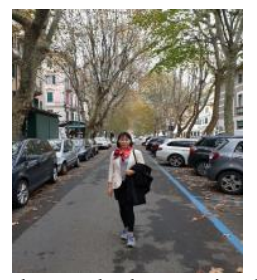

Budsaba Kanoksilapatham was born in Samut Sakhon, Thailand. She completed the bachelor's degree in English (Hons.) at the Faculty of Arts, Chulalongkorn University. Upon obtaining her master's degree in linguistics and EFL at Southern Illinois University at Carbondale (SIUC), USA, she joined the English Department, Faculty of Arts, Silpakorn University. She proceeded to study for her doctoral degree in linguistics at Georgetown University (GU), USA, completed her degree in linguistics (concentration: applied linguistics), and returned to work at Silpakorn University. While working on her dissertation in the USA, she was a part-time instructor at the English Language Center, University of Maryland at Baltimore County. She completed her dissertation, with Professor Swales and Professor Dr. Biber as her dissertation examiners.

Being passionate about discourse analysis, specifically genre analysis, and being determined to contribute to the betterment of English education of the entire paradigm in Thailand, the active engagement in academic activities including research projects and articles, textbooks, lectures and talks, and more than 40 keynote speeches both nationally and internationally has been a natural fil for her academic life. A number of books were written and a large number of research articles were published in prestigious international journals. Due to her distinguished contribution and dedication to the field, Professor Budsaba Kanoksilapatham was the first person in Thailand to receive the award of "TRF Senior Research Scholar in English" from the Thailand Research Fund (TRF) in 2016. In 2020, she received the award of "Distinguished Researcher of Silpakorn University." She was presented both of the awards in 2016 and 2020 by her Royal Highness Princess Maha Chaksri Sirindhorn. 